\title{
Mobile Magnetic Impurities in a Fermi Superfluid: A Route to Designer Molecules
}

\section{Citation}

Gopalakrishnan, Sarang, Colin V. Parker, and Eugene Demler. 2015. “Mobile Magnetic Impurities in a Fermi Superfluid: A Route to Designer Molecules." Physical Review Letters 114 (4). https:// doi.org/10.1103/physrevlett.114.045301.

\section{Permanent link}

http://nrs.harvard.edu/urn-3:HUL.InstRepos:41412207

\section{Terms of Use}

This article was downloaded from Harvard University's DASH repository, and is made available under the terms and conditions applicable to Other Posted Material, as set forth at http:// nrs.harvard.edu/urn-3:HUL.InstRepos:dash.current.terms-of-use\#LAA

\section{Share Your Story}

The Harvard community has made this article openly available.

Please share how this access benefits you. Submit a story.

Accessibility 


\title{
Mobile Magnetic Impurities in a Fermi Superfluid: A Route to Designer Molecules
}

\author{
Sarang Gopalakrishnan, ${ }^{1}$ Colin V. Parker, ${ }^{2}$ and Eugene Demler ${ }^{1}$ \\ ${ }^{1}$ Department of Physics, Harvard University, Cambridge, Massachusetts 02138, USA \\ ${ }^{2}$ James Franck Institute and Department of Physics, University of Chicago, Chicago, Illinois 60637, USA
}

(Received 4 June 2014; published 28 January 2015)

\begin{abstract}
A magnetic impurity in a fermionic superfluid hosts bound quasiparticle states known as Yu-ShibaRusinov states. We argue here that, if the impurity is mobile (i.e., has a finite mass), the impurity and its bound Yu-Shiba-Rusinov quasiparticle move together as a midgap molecule, which has an unusual "Mexican-hat" dispersion that is tunable via the fermion density. We map out the impurity dispersion, which consists of an "atomic" branch (in which the impurity is dressed by quasiparticle pairs) and a "molecular" branch (in which the impurity binds a quasiparticle). We discuss the experimental realization and detection of midgap Shiba molecules, focusing on Li-Cs mixtures, and comment on the prospects they offer for realizing exotic many-body states.
\end{abstract}

DOI: 10.1103/PhysRevLett.114.045301

PACS numbers: 67.85.Pq, 03.75.Ss, 74.62.En

A key project in ultracold atomic physics [1] involves using the richness of atomic structure to create "designer" many-body systems - e.g., spins with $S U(N)$ symmetry [2] or bosons in gauge fields [3] - that have no solid-state equivalent. Condensed matter physics, meanwhile, has developed the converse project of exploiting many-body correlations to generate quasiparticles (e.g., anyons [4]) that are qualitatively unlike electrons or atoms. Such quasiparticles are usually excitations, but might exist, even at zero temperature, at impurities, topological defects, or edges [5]. In real materials, impurities, edges, etc., are immobile on the time scales of interest. But ultracold atomic systems do not have this restriction, and in these systems impurities are naturally mobile; hence, the impurity and its captured quasiparticle can form a coherently moving molecule. Binding exotic quasiparticles to mobile impurities offers a new method for designing particles whose dispersion and exchange statistics are inherited from an underlying correlated many-body state. Such "designer molecules" can access regimes of few- and many-body physics that are inaccessible by purely atomic or solid-state approaches.

Here, we consider perhaps the simplest such system, comprising a mobile magnetic impurity in a fermionic superfluid; natural experimental realizations include twospecies mixtures (e.g., Li-Cs mixtures) in which one species is fermionic. When the impurity is spatially localized, it binds a midgap quasiparticle state, called a Yu-Shiba-Rusinov (YSR) state [6-17]. Depending on the impurity-fermion coupling, the YSR state is either occupied or empty at zero temperature. We argue that when the impurity is mobile, it moves together with its quasiparticle state, forming a midgap "Shiba" molecule; at strong coupling, this molecule is the ground state of the system (Fig. 1). The midgap Shiba molecule differs from the molecule formed by an impurity in a one-component Fermi gas [18-22]; the midgap Shiba molecule exists deep in the
BCS limit, where there are no two-body bound states. Furthermore, the midgap Shiba molecule has an unusual dispersion with a spherical minimum inherited from the Fermi surface (Fig. 1). Spherical dispersion minima have attracted interest in the context of light-induced Rashba spin-orbit
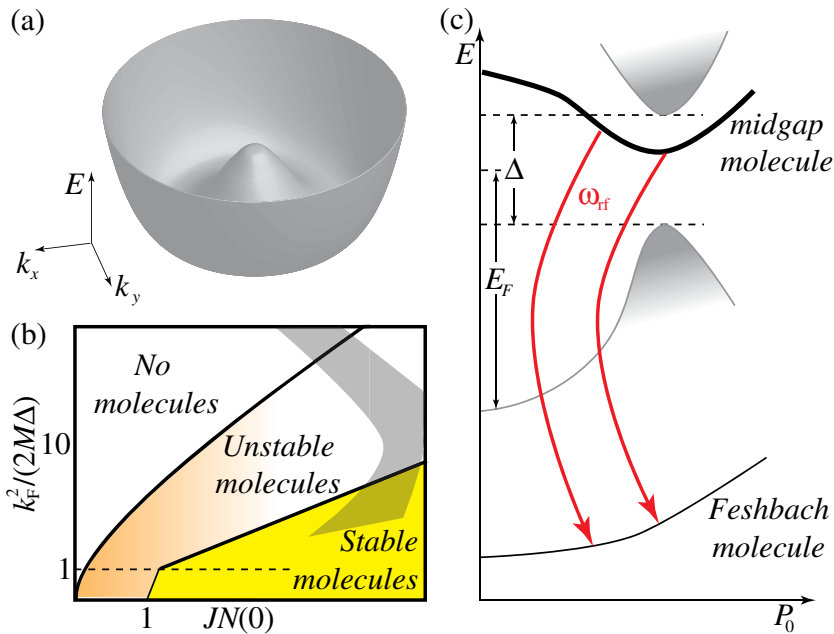

FIG. 1 (color online). (a) Mexican-hat dispersion of midgap Shiba molecule. (b) Phase diagram in three dimensions, as a function of impurity mass $M$ and impurity-fermion coupling $J$. As $J$ is increased, the system goes from a phase in which the midgap Shiba molecule does not exist, to one in which it exists as an excited (i.e., unstable) state, and finally one in which it is the ground state. Boundaries are given by Eqs. (4), (6) (including mass renormalizations as discussed in text). For heavy impurities (below dashed gray line), one recovers fixed-impurity behavior. Shaded region indicates the achievable parameter regime for $\mathrm{Li}$ Cs mixtures near the $843 \mathrm{G}$ and $880 \mathrm{G}$ heteronuclear resonances $[27,28]$. (c) Dispersion relation, showing midgap Shiba molecule (thick black line), "undersea" Feshbach molecule (thin black line), and Bogoliubov quasiparticles (gray lines). The midgap Shiba molecule's dispersion can be mapped out by driving radiofrequency (rf) transitions from it to the Feshbach molecule. 
coupling, because their high degeneracy enhances interactions, stabilizing exotic correlated phases [23-25]. Optically realizing an isotropic Rashba dispersion is challenging [26], whereas the dispersion of midgap Shiba molecules in an isotropic system is automatically isotropic.

The parameter controlling the midgap Shiba molecule's unusual properties is the impurity recoil energy, $\mathcal{E} \equiv 2 k_{F}^{2} / M$, where $M$ is the impurity mass and $k_{F}$ is the Fermi momentum [29]. For heavy impurities, $\mathcal{E}$ is small compared with the impurity-fermion coupling; therefore, impurity scattering mixes all the states near $k_{F}$, and the bound-state properties resemble those of a fixed impurity (Fig. 2). However, when $\mathcal{E}$ is large, processes scattering a quasiparticle across $2 k_{F}$ are off resonance by $\mathcal{E}$ [30]; therefore, the lowest-energy molecular-branch states consist of an impurity with momentum $\sim 0$ and a quasiparticle with momentum $\sim k_{F} \hat{\mathbf{n}}$ along some specific direction $\hat{\mathbf{n}}$. Consequently, when a molecule exists, it must have center-of-mass momentum $k_{F}$. Since $\hat{\mathbf{n}}$ is arbitrary, the molecular branch has a circular or spherical dispersion minimum by symmetry.

Below, we address the central questions concerning these unusual molecules and polarons. First, we identify critical couplings for the midgap Shiba molecule to exist as (a) an excited state, and (b) the ground state. Second, we compute the effective-mass corrections for both the impurity itself (the "polaron") and the midgap Shiba molecule, thus mapping out the full dispersion of the one-impurity problem. Finally, we discuss the regime of validity of our analysis, and propose an experimental method for probing the midgap Shiba molecule.

Model.-We consider a system governed by the Hamiltonian

$$
\begin{aligned}
\mathcal{H} & =\mathbf{P}^{2} /(2 M)+H_{\mathrm{BCS}}+H_{\mathrm{int}}, \\
H_{\mathrm{BCS}} & =\sum_{\mathbf{k}}\left[\sum_{\sigma} \epsilon_{k} c_{\mathbf{k} \sigma}^{\dagger} c_{\mathbf{k} \sigma}+\Delta\left(c_{\mathbf{k} \uparrow}^{\dagger} c_{-\mathbf{k} \downarrow}^{\dagger}+\text { H.c. }\right)\right], \\
H_{\mathrm{int}} & =\mathcal{V}^{-1} \sum_{\mathbf{k k}^{\prime} \sigma}(V+J \sigma) e^{i\left(\mathbf{k}-\mathbf{k}^{\prime}\right) \cdot \mathbf{x}}\left(c_{\mathbf{k} \sigma}^{\dagger} c_{\mathbf{k}^{\prime} \sigma}+\text { H.c. }\right) .
\end{aligned}
$$
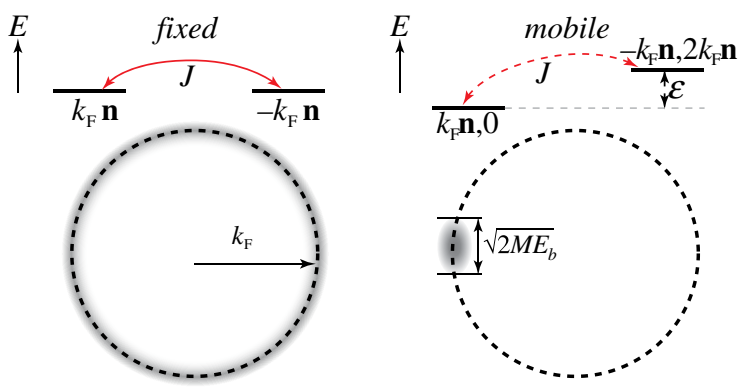

FIG. 2 (color online). Differences between bound states for a fixed impurity (left) and a mobile impurity (right). Scattering across the entire Fermi surface is resonant for the fixed impurity but off-resonant for the mobile impurity owing to recoil (top panel). Consequently, the Shiba state has contributions from all over the Fermi surface for a fixed impurity but only a small patch of the Fermi surface for a mobile impurity (lower panel).
Here, $\mathbf{X}, \mathbf{P}$ are the impurity position and momentum; $H_{\mathrm{BCS}}$ and $H_{\text {int }}$ are, respectively, the fermionic BCS Hamiltonian and the fermion-impurity interaction; $c_{\mathbf{k} \sigma}$ annihilates a microscopic fermion of momentum $\mathbf{k}$ and spin $\sigma= \pm 1$; $\epsilon_{k}=v_{F}\left(k-k_{F}\right)$ is the linearized free fermion dispersion; $\Delta$ is the superconducting gap; $V$ and $J$ are spin-independent and spin-dependent parts of the impurity-fermion interaction; and $\mathcal{V}$ is the system volume. In terms of Bogoliubov quasiparticle operators $\quad \gamma_{\mathbf{k} \uparrow} \equiv u_{\mathbf{k} \uparrow} c_{\mathbf{k} \uparrow}+v_{\mathbf{k} \uparrow} c_{-\mathbf{k} \downarrow}^{\dagger}, \quad$ one can rewrite $H_{\mathrm{BCS}}=\sum_{\mathbf{k} \sigma} E_{k} \gamma_{\mathbf{k} \sigma}^{\dagger} \gamma_{\mathbf{k} \sigma}$; the quasiparticle dispersion is $E_{k}=\sqrt{\Delta^{2}+\epsilon_{k}^{2}}$. Conserved quantities under $\mathcal{H}$ are (i) the total impurity plus fermion momentum $\mathbf{P}_{0}$, (ii) the fermion parity, and (iii) the number difference between $\uparrow$ and $\downarrow$ fermions. We focus on the experimentally relevant three-dimensional case; the one-dimensional case is discussed in the Supplemental Material [31].

Molecular threshold.-We first estimate the threshold for a molecular state to exist, using perturbation theory in $H_{\text {int }}$. We express $H_{\text {int }}$ in terms of quasiparticles; for simplicity we take $V=0$,

$$
\begin{aligned}
H_{\mathrm{int}}= & \mathcal{V}^{-1} \sum_{\mathbf{k} \mathbf{k}^{\prime} \sigma} e^{i\left(\mathbf{k}-\mathbf{k}^{\prime}\right) \cdot \mathbf{X}}\left[J \sigma\left(u_{\mathbf{k}} u_{\mathbf{k}^{\prime}}^{*}+v_{\mathbf{k}} v_{\mathbf{k}^{\prime}}^{*}\right)\right] \gamma_{\mathbf{k} \sigma}^{\dagger} \gamma_{\mathbf{k}^{\prime} \sigma} \\
& +\sum_{\mathbf{k} \mathbf{k}^{\prime}} e^{i\left(\mathbf{k}-\mathbf{k}^{\prime}\right) \cdot \mathbf{x}}\left[J \sigma u_{\mathbf{k}} v_{\mathbf{k}^{\prime}} \gamma_{\mathbf{k} \downarrow} \gamma_{-\mathbf{k}^{\prime} \uparrow}\right]+\text { H.c. }
\end{aligned}
$$

Perturbatively, we are only concerned with states for which $E_{\mathbf{k}} \simeq \Delta$; for these, $u_{\mathbf{k}} \approx v_{\mathbf{k}} \approx 1 / \sqrt{2}$ [32]. Here the $\gamma^{\dagger} \gamma$ terms involve scattering between the impurity and a quasiparticle; $\gamma^{\dagger} \gamma^{\dagger}(\gamma \gamma)$ terms create (destroy) quasiparticle pairs. Pair creation or destruction inevitably changes the energy by $\sim 2 \Delta$, and is off-resonant, whereas the energy change associated with scattering a quasiparticle from one state to another can be arbitrarily small. Therefore, to leading order, we neglect the second line of Eq. (2); under this approximation, the total quasiparticle number is conserved. We wish to look for a bound state of the impurity and one quasiparticle; evidently, this is a two-particle scattering problem with a contact interaction. The unusual feature is the Mexican-hat quasiparticle dispersion: the lowest-energy states with one quasiparticle are those in which the quasiparticle has momentum $\sim k_{F} \hat{\mathbf{n}}$, where $\hat{\mathbf{n}}$ is an arbitrary unit vector, and the impurity has momentum $\mathbf{P}=0$; thus, $\mathbf{P}_{0}=k_{F} \hat{\mathbf{n}}$. The perturbation couples such a state to other states with impurity momentum $\mathbf{p}$ and quasiparticle momentum $k_{F} \hat{\mathbf{n}}-\mathbf{p}$. Because states with $|\mathbf{p}| \simeq k_{F}$ are suppressed by large recoil energy denominators $\sim \mathcal{E}$, we assume $|\mathbf{p}| \ll k_{F}$. Taking $\hat{\mathbf{n}}=\hat{\mathbf{x}}$, the energy of a state with a given $\mathbf{p}$ is

$\Xi_{\mathbf{p}} \simeq \frac{\left(p^{2}\right)}{2 M}+\Delta+\frac{v_{F}^{2}}{2 \Delta} p_{x}^{2}=\Delta+\frac{p_{x}^{2}}{\left(\frac{1}{2 M}+\frac{v_{F}^{2}}{2 \Delta}\right)^{-1}}+\frac{p_{\perp}^{2}}{2 M}$,

where $p_{\perp} \equiv\left(p_{y}, p_{z}\right)$. This is simply the dispersion of a free particle with an anisotropic mass, $M_{y}=M_{z}=M$, 

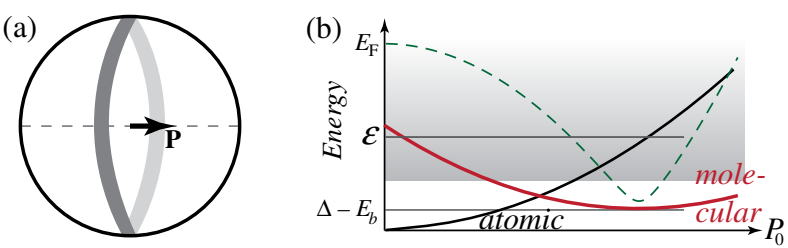

FIG. 3 (color online). (a) An impurity with momentum $\mathbf{P}$ is likeliest to create quasiparticle pairs along the shaded strip of the Fermi surface; these excitations, being tangent to the Fermi surface, are infinitely massive. (b) Schematic dispersions of the lowest states in the atomic (blue) and molecular (red) branches for weak coupling $J N(0) \simeq 0.2$; here, effective-mass corrections are small. For $\Delta \ll \mathcal{E}$, the branches cross. At high energies (shaded) these branches merge into the multiparticle continuum.

$M_{x}=\left(1 / M+v_{F}^{2} / \Delta\right)^{-1}$. Thus, the criterion for the midgap Shiba molecule to exist is the same as that for a particle with anisotropic mass, subject to an attractive contact potential, to have a bound state. Bound states correspond to zeroes of the inverse $T$-matrix [11], which takes the form $T^{-1}(\omega) \sim 1 / J-\sqrt{M_{x} M_{y} M_{z}}(A-B \omega)$, where $A, B$ are expressions that do not depend on the impurity mass but do in general depend on a high-energy cutoff. We eliminate this cutoff-dependence using our knowledge of the infinite-mass (i.e., pinned-impurity) bound state energy $E_{\infty} \simeq \Delta J^{2} N(0)^{2}$. We then find that the threshold $J_{0}$ for the molecular state to exist is

$$
J_{0} N(0) \simeq \frac{(2 m)^{3 / 2}}{M}\left(\frac{1}{M}+\frac{v_{F}^{2}}{\Delta}\right)^{1 / 2} \sqrt{\frac{E_{F}}{E_{\infty}}},
$$

whereas, for $|J|>J_{0}$, the molecular binding energy (measured from the gap edge) is

$$
E_{b} \simeq-E_{\infty}\left(1-J_{0} /|J|\right) .
$$

Here, $m$ is the fermion mass; $N(0) \sim m k_{F}$ is the density of states per unit volume at the Fermi level. Moreover, the relative-coordinate wave function of the molecule decays exponentially, with a characteristic real-space size of $1 / \sqrt{2 M\left|E_{b}\right|}$ in the directions tangent to the Fermi surface and $v_{F} / \sqrt{2 \Delta\left|E_{b}\right|}$ (generally much longer) in the normal direction.

The midgap Shiba molecule's dispersion follows analogously. Rotational invariance implies that there is a bound state of equal binding energy for every $\mathbf{P}_{0}$ whose magnitude is $k_{F}$. Thus, the molecule has a spherical dispersion minimum centered at $k_{F}$. The mass in the direction normal to the Fermi surface is simply the sum of the impurity mass and the inverse curvature of the quasiparticle dispersion, $M_{\text {mol. }}^{\perp}=M+\Delta / v_{F}^{2}$.

Parity-changing transition-For small $J$, the bound-state energy is close to the gap edge. Thus, the midgap Shiba molecule costs energy $\sim \Delta$ relative to the atomic-branch ground state (which has no quasiparticles). As $J$ increases, the gap between atomic and molecular branches closes, and they cross at some $J_{c}[33,34]$. In this regime, $\Delta$ cannot be treated as large; however, we retain the assumption that the recoil $\mathcal{E}$ is a large scale (Fig. 2). Specifically, we assume $J, \Delta \ll \mathcal{E} \ll E_{F}$. We then find the molecular energy by computing the $T$-matrix for impurity-quasiparticle scattering in the ladder approximation (see the Supplemental Material [31]). We find that the critical coupling obeys

$$
J_{c} \simeq\left[k_{F}^{2} /(M \Delta)\right] J_{c}^{\infty},
$$

where $J_{c}^{\infty} \sim 1 / N(0)$ is the fixed-impurity transition point [11]. The $M$-dependence follows from phase-space considerations. In the fixed-impurity limit, the bound state involves quasiparticle states from the entire Fermi surface (Fig. 2, left). By contrast, for a mobile impurity, recoil limits accessible quasiparticle states to a patch of transverse dimension $\sim \sqrt{M E_{b}}$. This phase-space reduction means the critical $J_{c}$ needed for a given bound-state energy is increased by a factor $\left(k_{F} / \sqrt{M E_{b}}\right)^{2}$ relative to the fixed impurity case.

Effective mass.-So far, we have explored the effects of the impurity recoil on the bound-state spectrum. We now discuss how the fermions affect the impurity recoil via polaronic effective-mass shifts [35], involving processes in which the impurity emits and reabsorbs virtual quasiparticle pairs. Because each quasiparticle pair costs an energy $\gtrsim 2 \Delta$, the creation of many pairs is suppressed (i.e., there is no orthogonality catastrophe). Moreover, for kinematic reasons, these pairs are likeliest to lie on the dispersion minimum [Fig. 3(a)]. Thus, the quasiparticle-pair energy $\approx 2 \Delta$, and the perturbative impurity energy shift is

$$
-J^{2}(m \Delta)^{3 / 2} \int d^{3} q /\left\{\frac{\mathbf{P}^{2}}{2 M}-\left[\frac{(\mathbf{P}-\mathbf{q})^{2}}{2 M}+2 \Delta\right]\right\} .
$$

The ultraviolet divergence in this expression can be eliminated by accounting for the high- $q$ behavior of the interaction vertex. For computing the effective mass, one need not regularize this divergence: the second derivative at $P=0$ converges, yielding the effective mass

$$
M^{*} \simeq M\left\{1+3 /\left(\sqrt{2} \pi^{4}\right) J^{2} \Delta(m M)^{3 / 2}\right\} .
$$

The effective mass of the midgap Shiba molecule (obtained similarly) is

$$
M_{\mathrm{mol}}^{\perp, *} \simeq M_{\mathrm{mol}}^{\perp}\left[1+1 /\left(16 \sqrt{2} \pi^{5}\right) J^{2} k_{F}^{2}\left(m^{3} M_{\mathrm{mol}}^{\perp}\right)^{1 / 2}\right] .
$$

For larger $J$, one must go beyond perturbation theory; as detailed in the Supplemental Material [31], one can find $M^{*}$ self-consistently by (a) replacing the bare interaction with the $T$-matrix [36], and (b) including processes in which the impurity emits multiple quasiparticle pairs. When $J N(0) \gg 1$, we find the $J$-independent result

$$
M^{*} \simeq m\left[E_{F}^{2} /\left\{\mathcal{E}\left(\Delta-E_{b}\right)\right\}\right]^{2 / 3} .
$$



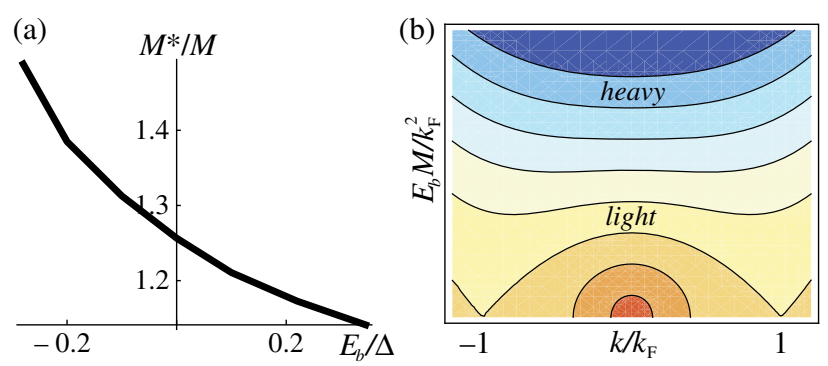

FIG. 4 (color online). (a) Effective mass in one dimension as a function of Shiba state energy, computed using the $T$-matrix approach (see the Supplemental Material [31]); $M^{*}$ evolves smoothly across the parity-changing transition at $E_{b}=0$. (b) Crossover from light impurities (two dispersion minima) to heavy impurities (single minimum).

The dressed impurity retains a Mexican-hat dispersion (and our calculations remain self-consistent) as long as $\mathcal{E} \gtrsim \sqrt{\Delta E_{F}}$, i.e., in the BCS limit. This result, although derived specifically for the Shiba molecule, is in fact a general result for polaron problems in which the "bath" dispersion is Mexican-hat-shaped (e.g., Rashba spin-orbit coupled systems [23]).

Combining results for $E_{b}$ and $M^{*}$, one can construct the full dispersion of the impurity [Fig. 3(b)]. At small $P_{0}$, the lowest-energy state is in the atomic branch with effective mass $M^{*}$; as $P_{0}$ increases, the atomic and molecular branches cross (provided that $\Delta \lesssim \mathcal{E}$ ), and the dispersion near $k_{F}$ is Mexican-hat shaped with a curvature $M_{\mathrm{mol}}^{\perp, *}$. At momenta $\left(2 M^{*} \Delta\right)^{1 / 2} \lesssim P_{0} \lesssim k_{F}-\left(M_{\text {mol }}^{\perp, *} \Delta\right)^{1 / 2}$, the impurity radiates into the two-quasiparticle continuum. As $J$ increases, the minimum of the molecular branch at $P_{0}=k_{F}$ decreases through zero, and the ground state changes via a first-order phase transition (states of different fermion parity cannot mix). Thus the parity transition discussed here resembles polaron transitions [37] in which the ground-state momentum changes abruptly.

Heavy-impurity limit.-We now discuss the crossover between "light" and "heavy" impurities. For simplicity, we work in one dimension; here, a Shiba state forms at each Fermi point. These states are mixed by an interaction matrix element $\sim E_{b}$; however, scattering across the Fermi surface costs $\sim \mathcal{E}$. This $2 \times 2$ Shiba subspace has the Hamiltonian

$$
\mathbb{H}=\left(\begin{array}{cc}
\frac{1}{2 M}\left(P_{0}-k_{F}\right)^{2}+E_{b} & E_{b} \\
E_{b} & \frac{1}{2 M}\left(P_{0}+k_{F}\right)^{2}+E_{b}
\end{array}\right) .
$$

The smallest eigenvalues of $\mathbb{H}$ occur for $P_{0} \simeq \pm k_{F}$ (the case discussed above) when the recoil is large; for a heavy impurity, however, the dispersion minimum moves to $P_{0}=0$ [Fig. 4(b)]. A similar crossover occurs in any dimension.

$B E C-B C S$ crossover.-The analysis above assumed $E_{F} \gg \Delta$; this is valid in the BCS limit. However, our qualitative conclusions are based on the observation that the quasiparticle dispersion has a minimum at some nonzero momentum $k_{0}$. This remains true in the unitary regime but with $k_{0}<k_{F}$; therefore, our main results (in particular, the Mexican-hat dispersion) should extend to this regime if $k_{F}$ is replaced with $k_{0}$. Deep in the BEC regime, the quasiparticle dispersion has a minimum at $k=0$; our results do not apply here.

Experimental implementation.-The system discussed here can be realized in two-species atomic mixtures in which at least one species is fermionic. A promising realization involves Li-Cs mixtures [27,28], in the magnetic-field range of 834-900 G. The Li atoms form a BCS superfluid, while the Cs-Li interaction can be tuned through various heteronuclear Feshbach resonances [38,39]. The impurity recoil $\mathcal{E} \approx E_{F} / 5$, while $\Delta \sim 0.01-0.1 E_{F}$ [40]. The molecular dispersion can be directly probed using momentum-resolved radio-frequency spectroscopy, as follows. Suppose the impurity-fermion scattering length is negative for $\uparrow$ fermions and positive for $\downarrow$ fermions. Then $\uparrow$ fermions form a Shiba state; moreover, a Feshbach molecule of the impurity and a $\downarrow$ fermion must exist. One can use a rf pulse to flip the spin state and drive transitions between the midgap Shiba molecule and the Feshbach molecule. Because the rf pulse is momentum conserving, one can map out the dispersion relation of the midgap Shiba molecules by measuring the momentum of the Feshbach molecules (through time-of-flight imaging) as a function of frequency $[41,42]$.

Outlook.-We have argued that a moving magnetic impurity in a Fermi superfluid can capture a quasiparticle and form an exotic midgap Shiba molecule with a Mexicanhat dispersion minimum; as this dispersion minimum maps out the Fermi surface, one can easily tune its shape by putting the fermions in an optical lattice. Depending on the impurity statistics, the molecule can be bosonic or fermionic. Moreover, we expect the intermolecular exchange interactions to be exotic. Qualitative aspects of these interactions can be deduced from recent work on pinned impurities $[43,44]$. Molecules interact by exchanging either continuum quasiparticles or Shiba states. Remarkably, for a moving impurity the Shiba-state exchange interaction is strongly angle-dependent because the molecular wave functions are anisotropic, as discussed above: molecules with center of mass momenta $\hat{\mathbf{n}}, \hat{\mathbf{n}}^{\prime}$ interact more strongly when $\hat{\mathbf{n}} \| \hat{\mathbf{n}}^{\prime}$ than when $\hat{\mathbf{n}} \perp \hat{\mathbf{n}}^{\prime}$. A quantitative treatment of these interactions will be given elsewhere. Such interactions make midgap Shiba molecules promising platforms to study the interplay between spherical dispersions and structured interactions. Note that a sufficiently high density of impurities might alter the character of the superfluid, favoring a modulated gap [45]. Finally, while we discussed impurities in $s$-wave superfluids, even more unusual properties might be realizable with impurities in unconventional (e.g., topologically paired) superfluids [13,46-48].

We thank Cheng Chin, Gergely Zarand, Brian Skinner, and Michael Knap for helpful discussions. This work was supported by the Harvard Quantum Optics Center (S. G.), 
the ARO-MURI Non-equilibrium Many-body Dynamics grant (C. V.P.), the Harvard-MIT Center for Ultracold Atoms, the DARPA OLE program, the AFOSR-MURI on New Quantum Phases of Matter, the ARO-MURI on Atomtronics, and the ARO MURI Quism program (E. D.).

[1] I. Bloch, J. Dalibard, and W. Zwerger, Rev. Mod. Phys. 80, 885 (2008).

[2] A. V. Gorshkov, M. Hermele, V. Gurarie, C. Xu, P. S. Julienne, J. Ye, P. Zoller, E. Demler, M. D. Lukin, and A. M. Rey, Nat. Phys. 6, 289 (2010).

[3] J. Dalibard, F. Gerbier, G. Juzeliūnas, and P. Öhberg, Rev. Mod. Phys. 83, 1523 (2011).

[4] H. L. Stormer, D. C. Tsui, and A. C. Gossard, Rev. Mod. Phys. 71, S298 (1999); C. Nayak, S. H. Simon, A. Stern, M. Freedman, and S. Das Sarma, Rev. Mod. Phys. 80, 1083 (2008).

[5] L. Fu and C. L. Kane, Phys. Rev. Lett. 100, 096407 (2008); R. M. Lutchyn, J. D. Sau, and S. Das Sarma, Phys. Rev. Lett. 105, 077001 (2010).

[6] L. Yu, Acta Phys. Sin. 21, 75 (1965).

[7] H. Shiba, Prog. Theor. Phys. 40, 435 (1968).

[8] A. I. Rusinov, Sov. Phys. JETP Lett. 9, 85 (1969).

[9] M. I. Salkola, A. V. Balatsky, and D. J. Scalapino, Phys. Rev. Lett. 77, 1841 (1996).

[10] A. Yazdani, B. Jones, C. Lutz, M. F. Crommie, and D. M. Eigler, Science 275, 1767 (1997).

[11] A. V. Balatsky, I. Vekhter, and J.-X. Zhu, Rev. Mod. Phys. 78, 373 (2006).

[12] E. Vernier, D. Pekker, M. W. Zwierlein, and E. Demler, Phys. Rev. A 83, 033619 (2011).

[13] H. Hu, L. Jiang, H. Pu, Y. Chen, and X.-J. Liu, Phys. Rev. Lett. 110, 020401 (2013); J. D. Sau and E. Demler, Phys. Rev. B 88, 205402 (2013).

[14] F. Pientka, L. I. Glazman, and F. von Oppen, Phys. Rev. B 88, 155420 (2013).

[15] S. Nadj-Perge, I. K. Drozdov, B. A. Bernevig, and A. Yazdani, Phys. Rev. B 88, 020407 (2013).

[16] J. Klinovaja, P. Stano, A. Yazdani, and D. Loss, Phys. Rev. Lett. 111, 186805 (2013).

[17] M. M. Vazifeh and M. Franz, Phys. Rev. Lett. 111, 206802 (2013).

[18] A. Schirotzek, C.-H. Wu, A. Sommer, and M. W. Zwierlein, Phys. Rev. Lett. 102, 230402 (2009).

[19] F. Chevy, Phys. Rev. A 74, 063628 (2006).

[20] R. Combescot, A. Recati, C. Lobo, and F. Chevy, Phys. Rev. Lett. 98, 180402 (2007).

[21] N. Prokof'ev and B. Svistunov, Phys. Rev. B 77, 020408 (2008).

[22] R. Combescot and S. Giraud, Phys. Rev. Lett. 101, 050404 (2008).

[23] T. D. Stanescu, B. Anderson, and V. Galitski, Phys. Rev. A 78, 023616 (2008); C. Wang, C. Gao, C.-M. Jian, and H. Zhai, Phys. Rev. Lett. 105, 160403 (2010); C. Wu, I. Mondragon-Shem, and X. F. Zhou, Chin. Phys. Lett. 28, 097102 (2011).

[24] C.-M. Jian and H. Zhai, Phys. Rev. B 84, 060508 (2011); S. Gopalakrishnan, A. Lamacraft, and P. M. Goldbart, Phys. Rev. A 84, 061604 (2011); T. Ozawa and G. Baym, Phys. Rev. A 85, 013612 (2012); R. Barnett, S. Powell, T. Graß,
M. Lewenstein, and S. Das Sarma, Phys. Rev. A 85, 023615 (2012); E. Berg, M. S. Rudner, and S. A. Kivelson, Phys. Rev. B 85, 035116 (2012); T. A. Sedrakyan, A. Kamenev, and L. I. Glazman, Phys. Rev. A 86, 063639 (2012); R. M. Wilson, B. M. Anderson, and C. W. Clark, Phys. Rev. Lett. 111, 185303 (2013); S. Gopalakrishnan, I. Martin, and E. A. Demler, Phys. Rev. Lett. 111, 185304 (2013).

[25] N. R. Cooper and J. Dalibard, Phys. Rev. Lett. 110, 185301 (2013).

[26] D. L. Campbell, G. Juzeliūnas, and I. B. Spielman, Phys. Rev. A 84, 025602 (2011); V. Galitski and I. Spielman, Nature (London) 494, 49 (2013).

[27] M. Repp, R. Pires, J. Ulmanis, R. Heck, E. D. Kuhnle, M. Weidemüller, and E. Tiemann, Phys. Rev. A 87, 010701 (2013).

[28] S.-K. Tung, C. Parker, J. Johansen, C. Chin, Y. Wang, and P. S. Julienne, Phys. Rev. A 87, 010702 (2013).

[29] Note that $\mathcal{E}$ is also the scale governing the physics of the so-called Kondo polaron [30].

[30] A. Lamacraft, Phys. Rev. Lett. 101, 225301 (2008); J. Rincón, D. J. García, K. Hallberg, and M. Vojta, Phys. Rev. B 88, 140407 (2013).

[31] See the Supplemental Material at http://link.aps.org/ supplemental/10.1103/PhysRevLett.114.045301 for details of the re-summed $T$-matrix approach and results.

[32] For pure potential scattering, the relevant coherence factor in the first line would be $u \mathbf{k} u_{\mathbf{k}^{\prime}}^{*}-v \mathbf{k} v_{\mathbf{k}^{\prime}}^{*}$, which vanishes for states near the gap, as expected from Anderson's theorem.

[33] M. I. Salkola, A. V. Balatsky, and J. R. Schrieffer, Phys. Rev. B 55, 12648 (1997).

[34] O. Sakai, Y. Shimizu, H. Shiba, and K. Satori, J. Phys. Soc. Jpn. 62, 3181 (1993).

[35] T. D. Lee, F. E. Low, and D. Pines, Phys. Rev. 90, 297 (1953).

[36] E. Braaten, M. Kusunoki, and D. Zhang, Ann. Phys. (Berlin) 323, 1770 (2008).

[37] V. M. Stojanović and M. Vanević, Phys. Rev. B 78, 214301 (2008); D. J. J. Marchand, G. De Filippis, V. Cataudella, M. Berciu, N. Nagaosa, N. V. Prokof'ev, A. S. Mishchenko, and P. C. E. Stamp, Phys. Rev. Lett. 105, 266605 (2010).

[38] M. Bartenstein, A. Altmeyer, S. Riedl, S. Jochim, C. Chin, J. H. Denschlag, and R. Grimm, Phys. Rev. Lett. 92, 120401 (2004).

[39] C. Chin, R. Grimm, P. Julienne, and E. Tiesinga, Rev. Mod. Phys. 82, 1225 (2010).

[40] C. Chin, M. Bartenstein, A. Altmeyer, S. Riedl, S. Jochim, J. H. Denschlag, and R. Grimm, Science 305, 1128 (2004).

[41] J. T. Stewart, J. P. Gaebler, and D. S. Jin, Nature (London) 454, 744 (2008).

[42] Q. Chen and K. Levin, Phys. Rev. Lett. 102, 190402 (2009).

[43] N. Yao, L. I. Glazman, E. A. Demler, M. D. Lukin, and J. D. Sau, Phys. Rev. Lett. 113, 087202 (2014).

[44] N. Y. Yao, C. P. Moca, I. Weymann, J. D. Sau, M. D. Lukin, E. A. Demler, and G. Zaránd, Phys. Rev. B 90, 241108(R) (2014).

[45] I. Zapata, B. Wunsch, N. T. Zinner, and E. Demler, Phys. Rev. Lett. 105, 095301 (2010); A. V. Rozhkov and D. P. Arovas, Phys. Rev. Lett. 82, 2788 (1999).

[46] V. Gurarie, L. Radzihovsky, and A. V. Andreev, Phys. Rev. Lett. 94, 230403 (2005).

[47] M. Sato, Y. Takahashi, and S. Fujimoto, Phys. Rev. Lett. 103, 020401 (2009).

[48] X.-L. Qi and S.-C. Zhang, Rev. Mod. Phys. 83, 1057 (2011). 